\title{
Experimental system for continuous monitoring of overhead power lines and substations insulation
}

\author{
Damir Zaripov ${ }^{1, *}$, and Rustem Nasibullin ${ }^{2}$ \\ ${ }^{1}$ Kazan State Energy University, Kazan, Russia \\ ${ }^{2}$ PJSC "Krasnogorskij Zavod named S.A. Zverev", Krasnogorsk, Russia
}

\begin{abstract}
This paper describes a system for monitoring the insulation condition of overhead power lines and substations based on sensors installed on towers and insulators. The sensors works on the capacitive coupling with an insulating structure and register the appearance and growing of electrical discharges near the insulators. The data from the sensors with a predetermined frequency (from 1 minute or more) are transmitted throw cellular communication to the operating service computer (smartphone) and presents as changing graphs on the screen. In this paper in operation and functional diagram of the system are proposed with described results of tests and experiments with manufactured product samples in laboratory and field conditions on an operating $110 \mathrm{kV}$ line. The results of field experiments shows the advantages of the system to monitor the insulation in comparing with traditional diagnostic devices.
\end{abstract}

\section{Introduction}

In the previous works in the middle of the last century (see, for example, [1]), devoted to diagnosing the insulation of high-voltage equipment, based on the analysis of experimental data and calculations, it was shown that the identification of defects in insulators is significantly influenced by external weather conditions and pollution. At the same time, it was noted that it would be possible to achieve significant results in diagnosing external insulation by organizing continuous monitoring under operating voltage. In those years, it was technically and economically difficult to implement it.

In recent year due to significant advances in microelectronics and wireless communication developing direction control elements of high voltage equipment based on sensor systems for continuous monitoring data transmission at radio frequencies (RF Sensor) [2], installed on insulating structures.

If we exclude manufacturing defects, internal and external overvoltages, then damage or overlapping of insulators in most cases is caused by long-term partial discharge (PD) activity on the insulation due to damage, pollution and moisture. If the insulation is in good condition, the PD should be practically absent on it. Therefore, the appearance and growth of PD intensity can serve as a criterion for assessing the state of insulation under continuous monitoring. With periodic diagnostics, for example, with UV cameras, it is almost impossible to track the tendency of insulation degradation. Detectability of insulation defects during periodic monitoring depends significantly on the weather during the work. Therefore, continuous monitoring of insulation condition has an advantage compared to the traditional monitoring, based on the periodic using the remote diagnostic devices.

Special place takes important role on the insulators overlap "unknown causes", occurring usually in the night and morning hours in light winds and without rainfall. The trigger for overlapping insulation can be: light pollution; high values of relative air humidity, about $91 \%$; jets of bird droppings [3] or rusty water flowing down from the traverse into the insulation [4]. The most likely reason for the frequent shutdowns of overhead power lines (OPL) at night and in the morning has not yet been established. Research is underway [5-7].

In recent days, there is a significant amount of development dedicated online systems for monitoring the insulation of OPL and substations, based on the registration of leakage current and the $\mathrm{PD}$, for example set out in Articles [8-11]. In the present work describing monitoring systems controlling overhead power lines (SCOPL) by signals PD based on sensors capacitive type, installed close to isolating structure.

\section{Structure of the insulation monitoring system}

The SCOPL consists of sensors, a hub and a smartphone (Fig. 1,2). Sensors mounted on the insulating structures OPL or near to the tower, transmit data with predetermined time intervals, and a hub mounted on one of the overhead towers, to give data from the sensors, transmits them by radio GSM standard service lines (Fig. 3 ). The smartphone system visualizes the received data in the form of changes graphs in the electric field strength on the insulator and the PD intensity in relative units (from 0 to 256), and also immediately signaling the insulation overlap (short circuit signal). The frequency of data updating can be set from the few seconds to several hours.

Transmitting devices SCOPL includes standard elements. The sensors transmit data to the hab at a frequency of $868 \mathrm{MHz}$, and those, in turn, work like a regular cellular phone with a SIM card. The sensor's device is original.

\section{Sensor's device}

The SCOPL sensor includes an electrode, a rectifier, a pulse shaping unit, a microcontroller, a wireless transmitter and a power supply unit (Fig. 4) [12]. All nodes are located on one printed circuit board, including the electrode, which is made in the form of an arc-shaped

\footnotetext{
Corresponding author: dzaripov@list.ru
} 
metallization layer. This device is an improvement of the early works of the authors $[13,14]$.

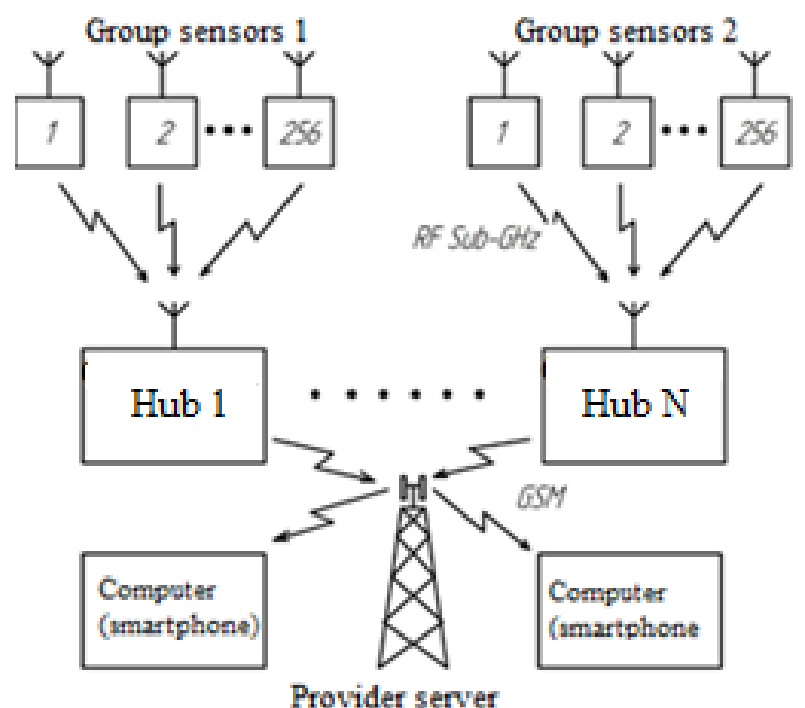

Fig. 1. Structure of SCOPL.

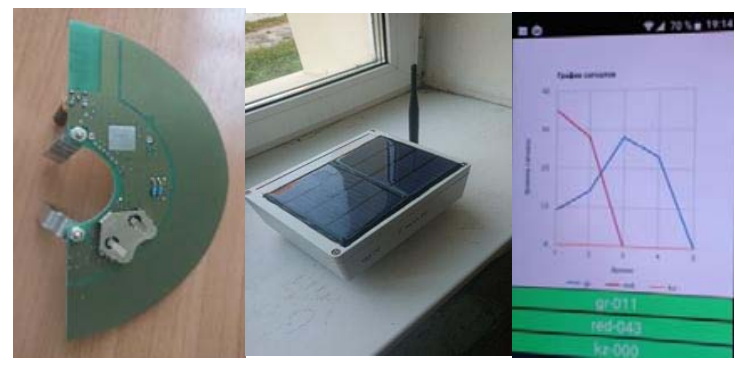

Fig. 2. Appearance of the sensor, hub and graphs on a smartphone.

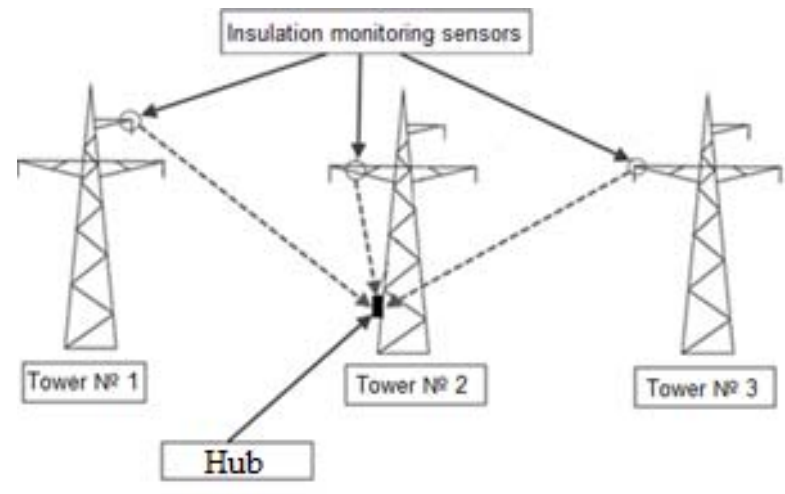

Fig. 3. Location of sensors and hub on OPL.

The electrode, which serves as a capacitor lining, forms a capacitor together with the high-voltage wire of the overhead line. It is connected to the overhead line support through the measuring part of the sensor. In existing of an alternating electric field on the power line, a current begins to flow through the electrode (El), which, passing through the sensor, is rectifying and converting into electrical pulses in the pulse shaping unit (PSU). The pulse frequency is proportional to the amount of current. To provide the required supply voltage and maintain this voltage in the event of a short-term surge of current when the wireless transmitter (WT) is turned on, a power supply (PS) unit with a $3 \mathrm{~V}$ battery is used. The microcontroller (MC) counts the pulses and controls their transmission.

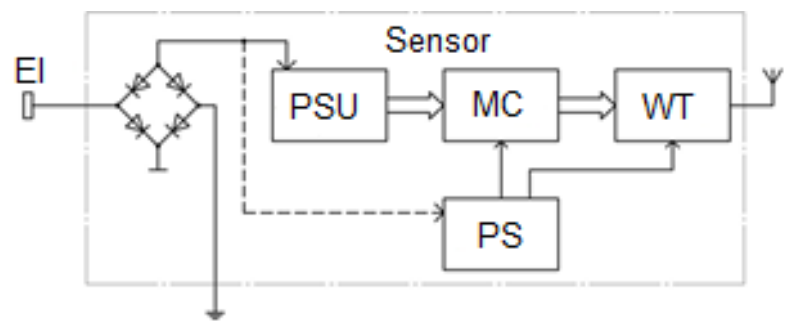

Fig. 4. Functional diagram of the sensor

The wireless transmitter transfer signals proportional to the pulse repetition rate at a given interval, as well as an immediate short-circuit signal when a corresponding pulse arrives at the input, which makes it possible to monitor the operation and fix the overlap of the isolating structure to the operator in real time.

It takes important role to determine the feasibility of this monitoring system is the battery life. The sensors must be functional for at least several years in adequate cost. The aim of the selection circuitry for sensors SCOPL is elk providing data at a predetermined interval while minimizing the energy consumption of the device.

The device with a diagram which is shown in Fig. 5 works when the insulation is in good condition or the defect is insignificant and there are no PD on the insulation, then the sensor detects signals from the electric field created by the voltage on the overhead line (LF signals). In achievement during the charging of the capacitor $\mathrm{C} 1$ on it opening the threshold voltage of the electronic key $(30-50 \mathrm{~V})$, the discharge occurs through its first pulse shaper 4 , causing pulse in the first pulse signal recorder inlet 7 . When this capacitor voltage drops to lower threshold, the electronic key stops conducting current and the capacitor $\mathrm{C} 1$ starts charging again, then cycle repeats. The frequency rate at the input of the recorder is periodic and depends on the EMF at the electrode sensor, which determines by the potential distribution along the insulating structure and they change when it is damaged or contaminated. The output of pulse recorder 7 continuously generates signals proportional to the pulse repetition rate at the input and transmits them to the wireless data transmitter 9 to send it to a smartphone. The elements of the circuit R1 and C2 are selected so that low-frequency oscillations of the network do not penetrate the second pulse shaper 5. In addition, due to the voltage drop across the valve 6 , the threshold voltage for switching on the electronic key of the second pulse shaper 5 is always higher and work only the first shaper pulse shaper 4.

Another situation, when the insulation is significantly damaged or contaminated and the intensity of the partial electrical discharges is high. PD create voltage pulses at the sensor input with a duration many orders of magnitude less than the period of the power frequency voltage (HF signals). At a certain value of these pulses, the input voltage the second shaper of pulses 5 with an electronic key, due to the low resistance of the capacitor C2 with high-frequency pulses, can exceed the voltage on the shaper 4 and then it will turn on and form a pulse first. At a high intensity of electrical discharges, the recorder 7 will receive only pulses to the second input from the shaper 5 . 


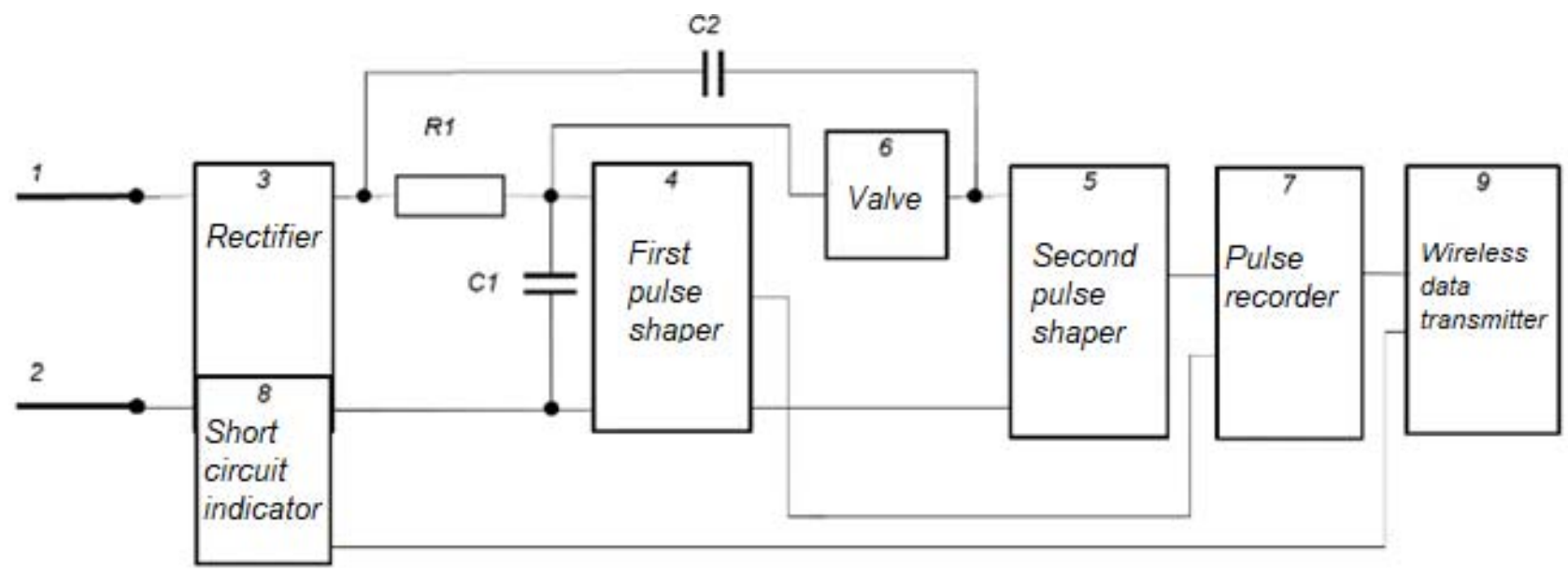

Fig. 5. Sensor circuit used to reveal the principle of its operation

The nature of the pulses is non-periodic and at high PD intensity it turns into continuous. Due to the presence of gate 6 , the pulses caused by discharges will not affect the operation of the first shaper 5 .

The average degree of insulation damage, when the intensity of electrical discharges is low, will be characterized by the random appearance of pulses from the second shaper 5 against the background of the pulses from the first (4). The threshold and frequency of occurrence of pulses from the second shaper will be determined by the time constant of the circuit of the second capacitor (C2) and this shaper (5), which together form a high-frequency filter.

The input of the wireless data transmitter 9 from the recorder 7 can continuously receive two groups of signals proportional to the pulse repetition rates from the shapers 4 and 5. The third signal at the input of the transmitter 9 will appear from the short circuit indicator 8 when the set threshold voltage at the input is exceeded. The short circuit signaling device is configured to fix the strong magnetic fields that occur when the electric arc overlaps the insulating structure on the tower.

In contrast to similar sensors, which usually measure the leakage current, the proposed device generates a signal proportional to the pulse repetition rate, which simplifies circuitry (no signal digitization is required), reduces power consumption and increases noise immunity.

\section{Experimental research}

According to the scheme described above were made experimental SCOPL, which was tested in high-voltage laboratory KGEU and OPL. In the laboratory the sensors were installed at different distances from the suspended insulator LK70/110 (Fig. 6). A voltage from 46 to $65 \mathrm{kV}$ was applied to the insulator. Simultaneously with the recording of data from the sensors, measurements of the leakage current were made using a TDS2000 oscilloscope connected to a $1 \mathrm{k} \Omega$ resistor installed in the cut between the insulator and ground. In total, three series of experiments were carried out:

- on a dry insulator;

- on an insulator moistened with tap water from a spray;
- on an insulator with an artificial defect in the form of a spark gap shunting several insulator skirts first from the high-voltage wire.

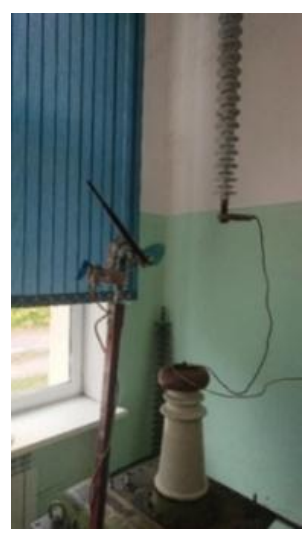

Fig. 6. Sensor on a test rig.

The signals from the sensors were recorded by SMS messages coming to the smartphone. In Fig. 7 shows graphs of signals, received from smartphone at various states of the insulator. In Fig. 8 shows the graphs of the leakage current $\left(\mathrm{I}_{\mathrm{lc}}\right)$. As expected, spraying water into the polymer surface did not significantly affect the average leakage current, but the discharge activity increased. The presence of a defect and is easily detected and sensors, and to measure the leakage current.

For further testing, the SCOPL, consisting of several sensors and a concentrator, was mounted on the anchor tower of the $110 \mathrm{kV}$ high-voltage line "AbdrakhmanovoUzlovaya" of Almetyevsk electrical networks, where polymer insulators were hung. One of the sensors was installed on the insulator terminations during scheduled power outage. The rest were mounted on the corners of the support and traverse ah near the controlled insulators without disconnecting the voltage on the overhead line. Signals were transmitted to the smartphone at $20 \mathrm{~min}$ intervals.

The choice of the location of the sensors on the support was determined by the possibility of their installation without removing the voltage so that the signals from the locations of the insulators exceeded the detection threshold. It is enough to install sensors at the edges of the traverse at the point of their attachment to the base of the support. 


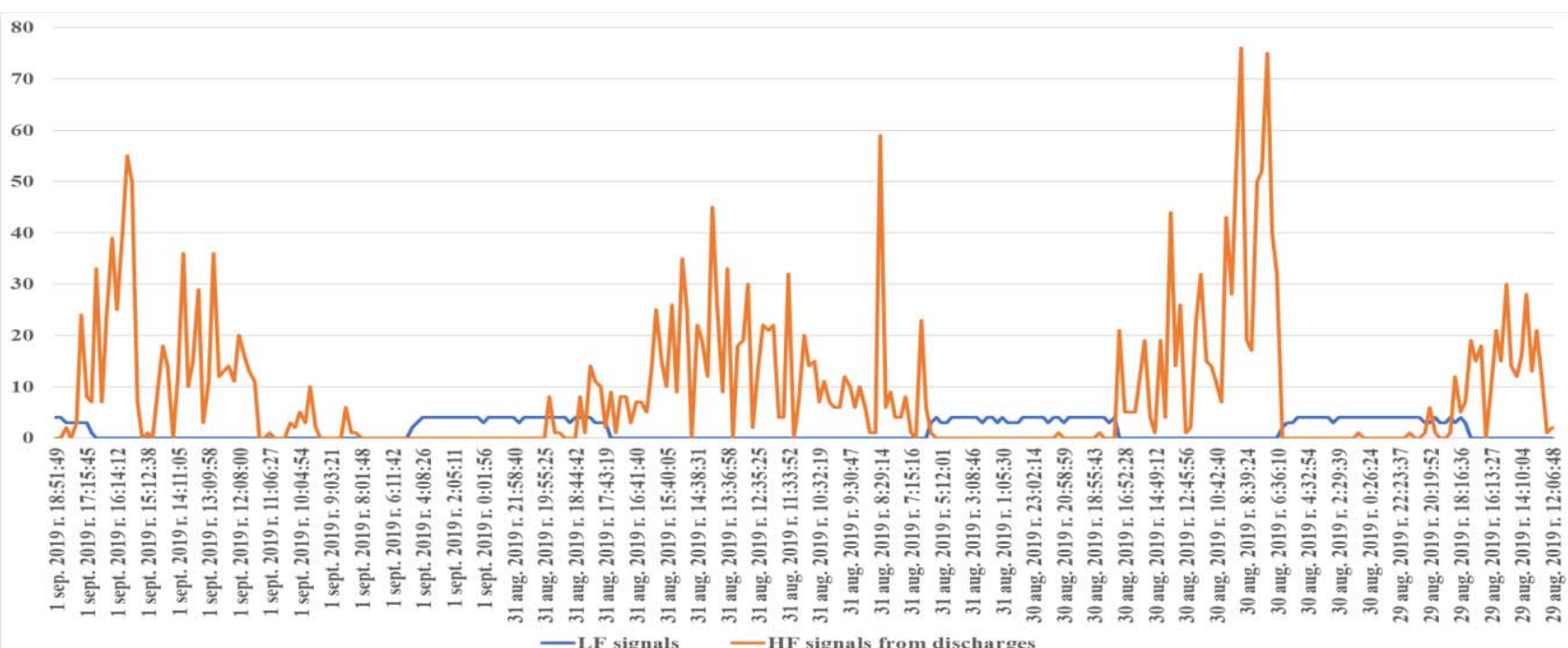

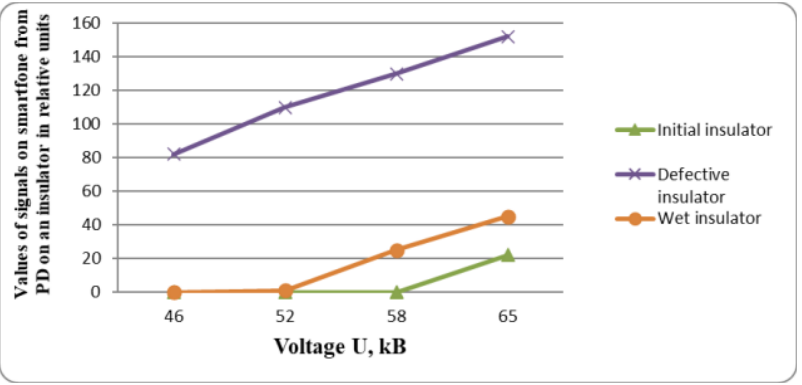

Fig. 7. Values of signals on a smartphone from $P D$ on an insulator.

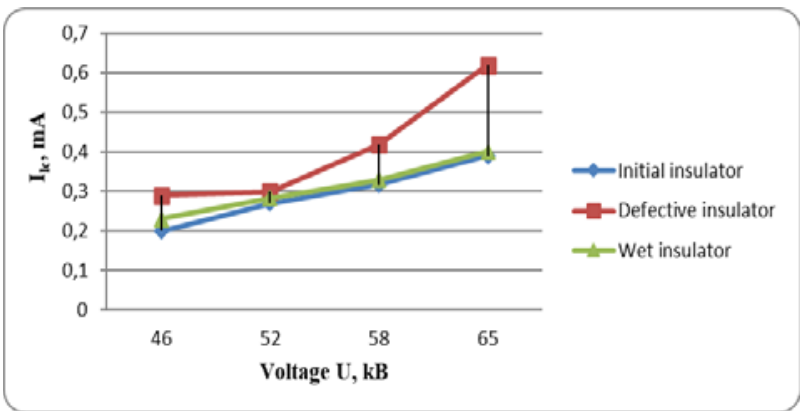

Fig. 8. The leakage current (RMS) on the insulator

During the operation of the sensors for several months in the summer and autumn period, signals about insignificant discharge activity on polymer insulators were repeatedly received on the smartphone. This mainly happened in bad weather. The situation that occurred at the end of August and the beginning of September stands out from this series, when signals about intense PDs appeared on the sensor, located on the side of the polymer insulator of the lower phase, which appeared early in the morning and subsided by the end of the day (see the graph in Fig. 9). Presumably, this activity was due to fog.

\section{Conclusion}

The presented system SCOPL may be mentioned the development of remote periodic diagnosis devices (UV camera, IR camera, ultrasound defectoscope), only metering $\mathrm{s}$ are performed much more frequently. In considering change of insulation condition on the external environment developed system is more informative.
Fig. 9. Graphs of signals on a smartphone from a sensor located on the overhead line support from the side of the phase a insulator, in relative units $(08 / 29 / 2019-09 / 01 / 2019)$

The obtained first results of the study of SCOPL allow us to hope for the possibility of its practical application today to identify sections of lines or individual supports where overlap or breakdown of insulation is most likely.

Improvement and reliability system operation is necessary for full works this system in lines.

\section{References}

1. Swee P.M. High-voltage insulation control by partial discharge method. Moscow: Energy, 1962. $106 \mathrm{p}$

2. A. Phillips, D. Mcguire, C. Engelbrecht. B3-301, CIGRE (2016)

3. R. Arbuzov, A. Ovsyannikov, News of electrical engineering, 4 (2008)

4. D. Hoch, N. Mahatho, F. Bolonga, URL https://www.researchgate.net/publication/2555 70809 WATER INDUCED DISCHARGES ON TRANSMISSION_VOLTAGE_SILICONE_RUBB ER INSULATORS (2006)

5. Borovitsky V.G. Study of the causes of unmotivated shutdowns of $110 \mathrm{kV}$ overhead lines and the development of recommendations for reducing their number: Dis. Cand. Tech. Sciences. Novosibirsk, 2013, $201 \mathrm{p}$.

6. "Research Provided Insight into Unexplained Line Outages", URL http://www.inmr.com/researchprovided-insight-unexplained-line-outages/ (2019)

7. "Learning from Service Experience with Composite Line Insulators",

URL https://www.inmr.com/learning-from-serviceexperience-with-composite-line-insulators-2/ (2020)

8. A. Levinzon, D. Kottick, R. Knijnik, L. Frenkel, B2205, CIGRE (2012)

9. X. Huang, Q. Sun, J. Ding, 3rd IEEE Conference on Industrial Electronics and Applications, Singapore, pp. 891-896 (2008)

10. A. Phillips, F. Bologna, J. Major, C. Engelbrecht, Proceedings of the 16th International Symposium on 
High Voltage Engineering, Cape Town, South Africa, paper 5-3, pp. 1-6

11. S. Campello Oliveira, E. Fontana, R. Bandeira Lima, IEEE Transactions Power Delivery, 24, 4, pp. 2257 2260 (2009)

12. Pat. RF 2731169 "Sensor for insulating structures condition continuous monitoring system", Zaripov D.K., Nasibullin R.A., Ibragimov R.R., Igtisamov M.Ch., priority of 30.12.2019 (2020)

13. RF Pat. 2517776 "Optical remote diagnostics method for insulating structure", priority of 03.12.2012, Zaripov D.K., (2014)

14. RF Pat. 2660754 "Light indicator of the state of the insulating structure state", priority of 14.07.2017, Zaripov D.K., Nasibullin R.A., Margulis S.M., Ibragimov R.R., Igtisamov M.Ch., (2018) 